\title{
First Experiences using Electron Holography with a Cs-Corrected TEM
}

\author{
Michael Lehmann, Dorin Geiger, Hannes Lichte \\ Dresden University, Triebenberg Laboratory, D-01062 Dresden, Germany
}

Conventional high-resolution transmission electron microscopy (HRTEM) suffers from the loss of phase information during image acquisition, which prevents an a-posteriori correction of coherent aberrations. This is also true for micrographs recorded by means of a Cs-corrected TEM. Despite the fact that the Cs-corrector corrects all coherent aberrations up to the third order, residual aberrations cannot be completely excluded. Consequently, a combination of Cs-corrected TEM with a holographic recording technique like focal series reconstruction [1] or off-axis electron holography [2] is most advantageous, which allows an a-posteriori correction of residual aberrations.

For off-axis electron holography, Cs-correction is very attractive, because the information limit is pushed to higher spatial frequencies, only limited by the chromatic envelope function. Moreover, the object exit-wave phase information is available without transfer gaps from large area phase modulations, e.g. by electric potentials and magnetic fields, up to atomic dimensions. The slight correction of coherent aberrations can be performed more accurately due to the considerably smoother dependence of the residual wave aberration from spatial frequency. The corresponding point-spread-function is virtually zero; hence the hologram width can be reduced to the width of the area under investigation gaining a higher dose of coherent electrons, which in turn improves the phase detection limit.

Here, first experiences are reported using a FEI Tecnai F20 TEM with Cs-corrector [3] (CEOS company), which is additionally equipped with a Möllenstedt biprism installed for electron holography in the SAD aperture holder. Compared with a Philips CM200FEG ST, the combination of SuperTWIN objective lens with Cs-corrector results in an about 1.2-times higher magnification at the first intermediate image plane yielding an advantageous geometric setup for the electron biprism. The resulting interference contrast is about 5-times better, which has as consequence a tremendous improvement of the phase detection limit. Figure 1 shows an off-axis electron hologram of a wedgeshaped GaAs-crystal in [110]-orientation. The interference fringe spacing is $0.07 \mathrm{~nm}$ at a biprism voltage of $U_{F}=300 \mathrm{~V}$ yielding a clear separation of the sidebands, i.e. the Fourier transform of the image wave, from the centerband. The interference fringe contrast is about $14.5 \%$. Due to residual aberrations, the reconstructed image wave (fig. 2) does not show the expected dumbbell-contrast of [110]-oriented GaAs. By minimizing the amplitude contrast of the thin amorphous edge, the residual coherent aberrations have been determined and correspondingly corrected yielding the expected dumbbell-contrast in amplitude and phase as shown in fig. 3. [4]

References:

[1] W.M.J. Coene, A. Thust, M. Op de Beeck, D. Van Dyck, Ultramicr. 64 (1996) 109-135.

[2] H. Lichte, Advances in Optical and Electron Microscopy 12 (1991) 25-91.

[3] M. Haider, H. Rose, St. Uhlemann, E. Schwan, B. Kabius, K. Urban, Ultramicr. 75 (1998) 53-60.

[4] Many thanks are due Dr. Peer Kruse from the University of Karlsruhe for providing the wedge-shaped GaAs sample. The Cs-corrected Tecnai F20 installed at the Triebenberg lab is a joint project of FEI, CEOS and TU Dresden. The financial support by the German Research Foundation (DFG) is gratefully acknowledged. 


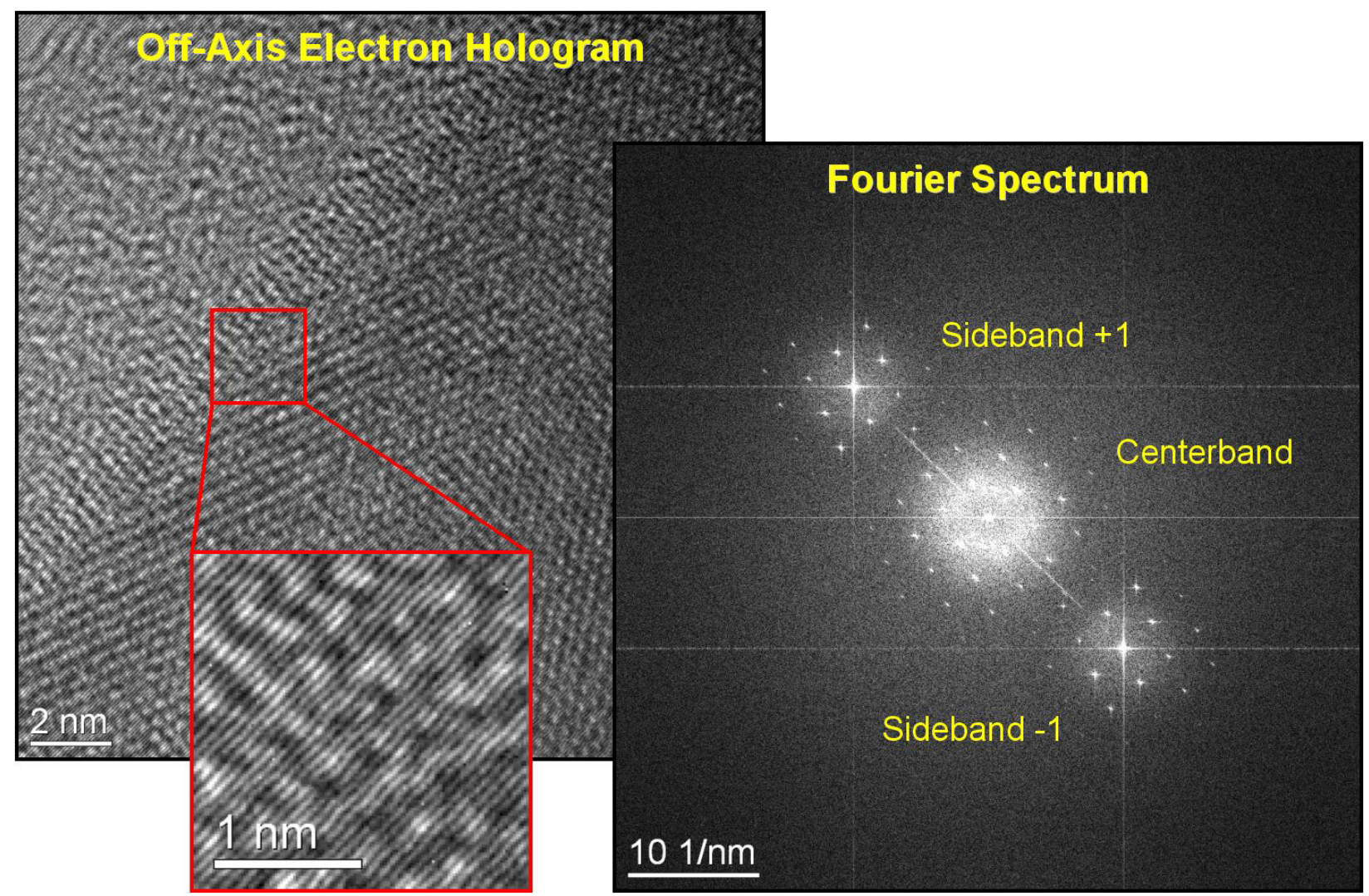

Fig. 1. Off-axis electron hologram of a wedge-shaped [110]-oriented GaAs-crystal. The magnified area shows the fine interference fringes with $0.07 \mathrm{~nm}$ spacing. Therefore, a clear separation of centerband and sidebands can be observed in the Fourier spectrum.
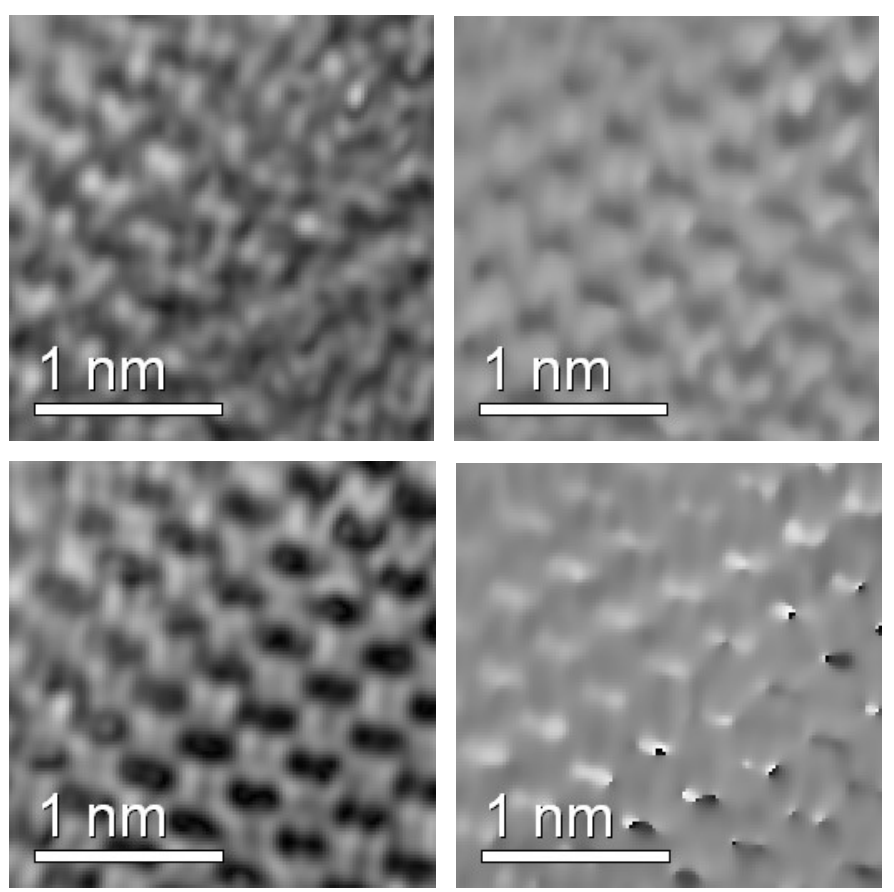

Fig. 2. Reconstructed image wave (amplitude: left; phase: right) from the magnified area (slightly noise reduced by Bragg-filtering). Due to residual aberrations, the atom column positions cannot be identified.

Fig. 3. Object exit-wave (amplitude: left; phase: right) as corrected for residual aberrations $\left(A_{1}=20 \mathrm{~nm}, \alpha_{A 1}=68^{\circ}\right.$, $C_{1}=14 \mathrm{~nm}, B_{2}=0.5 \mu \mathrm{m}, \alpha_{B 2}=280^{\circ}$, $C_{3}=-20 \mu \mathrm{m}$, slightly noise reduced by Bragg-filtering). The correct dumbbellcontrast can be observed in amplitude and phase. 